\title{
Optimal Resource Allocation of Virtual Organizations - The Perspective of Changeable Parameters
}

\author{
Jih-Jeng Huang ${ }^{+}$ \\ Department of Computer Science \& Information Management, Soochow University, Taiwan
}

\begin{abstract}
Virtual organizations (VOs) are flexible organizations, dynamically restructured from components of existing organizations and frequently created to capture the value of a market opportunity. That is, several organizations may join to form a VO and each organization has its own resource and capability. Hence, compared with other traditional organizations, the resource allocation of VOs is more complicated, because of the changeability of organizational parameters, such as, human resource, production resource, and technological capability. Hence, in this paper, three kinds of multiple objective programming (MOP) models with the concept of the changeable parameters are proposed to deal with the resource allocation problem of VOs.
\end{abstract}

Keywords: virtual organizations, resource allocation, multiple objective programming (MOP), changeable parameters.

\section{Introduction}

Virtual organizations (VOs) are defined as flexible organizations, composed of existing organizations for creating competitive advantages, to capture the value of a market opportunity (Shao and Liao, 1996). The characteristics of VOs are quick assembly and disassembly, and also by sharing of components among multiple organizations (Camarintha-Matos et al., 2005) to quickly response the needs of customers. To utilize the advantages of VOs, resource allocation between joined organizations plays a key role. The reason is that different organizations join to a VO for the complement resources between organizations. Hence, a VO only can create the competitive advantages if they use the joined resources well. Note that the virtuality is a matter of degree, since VOs may utilize some existing resources, such as employees and machines (Orman, 2009).

Traditionally, mathematical programming is widely used for the optimality of resource allocation. Many models, such as 0-1 programming (Luo and Zhao, 2009), integer programming (Donovan and Rideout, 2003) or dynamic programming (Powell and van Roy, 2004), are used for dealing with these problems. However, traditional methods are not suitable for dealing with the resource-allocation problems of VOs. First, compared with traditional organizations, the resources of VOs can be changeable and restructured by different organizations. Next, the purpose of VOs is to achieve or obtain the specific target of the market. However, traditional organizations simply optimize their system in given conditions.

Therefore, the purpose of this paper is to develop the possible models for VOs to optimize their systems. The major feature of our models is that all parameters of the system are assumed to be changeable. This feature enables VOs to deal with different kinds of resource allocation problems in a more realistic way. In addition, we propose three numerical examples to demonstrate the proposed models and compare the results with other methods. The results show that the proposed method can provide a flexible way for VOs to manage and optimize their resources to achieve the desired situation.

+ Corresponding author. Tel.: +88623111531.

E-mail address: jjhuang@ @cu.edu.tw. 


\section{MOP with changeable parameters}

In practice, there are three basic ways for a VO to possibly achieve its desired point: (1) increasing budgets, (2) improving objective coefficients, and (3) upgrading production efficiency. Next, we will discuss each situation and develop the corresponding optimal model as follows. In the first situation, a VO can make some financing decisions, e.g., to raise/borrow memory from banks/joined organizations or to issue company bonds/stocks in the capital market, etc. Then, the problem of achieving the desired point is equivalent to minimizing the extra budget under given objectives space and decision space (constraints).

Assume a VO has $n$ objectives to be achieved and $m$ products are produced. We can incorporate the concepts of financing decisions into MOP and formulate the following model:

<Model 1: MOP with changeable budgets>

$$
\begin{gathered}
\min \widehat{B} \\
\text { s.t. } \quad \sum_{j=1}^{m} c_{i j} x_{i j} \geq f_{i}^{* *}(\boldsymbol{x}), \quad i=1, \ldots, n, \\
\boldsymbol{p}^{\prime} \boldsymbol{A} \boldsymbol{x} \leq B+\widehat{B},<\text { extra conditions for } \widehat{B}> \\
\boldsymbol{x} \geq \mathbf{0},
\end{gathered}
$$

where $c_{i j}$ denotes the $j$ th coefficient of the $i$ th objective function, $f_{i}^{* *}(\boldsymbol{x})$ denotes the desired value of the $i$ th objective, $\boldsymbol{p}$ denotes the unit price vector of resources, $B$ is the original budget and $\widehat{B}$ denotes the extra budget obtained from financing decisions.

Example 1. Assume a VO produces two different products, suits and dresses, in quantities $x$ and $y$. Each of them costs five different resources, nylon through golden thread, according to technologically determined requirements. Unit prices of resources are also given, as shown in Table 1. If two objectives, namely profit $\left(f_{1}=400 x_{1}+300 x_{2}\right)$ and quality index $\left(f_{2}=6 x_{1}+8 x_{2}\right)$, are considered by the company. If the objective functions and constraints are constant, we can obtain the optimal solution from De Novo programming as $f_{1}=2375$ and $f_{2}=44.5$. However, the decision maker feels unsatisfactory with that results and hope to increase $f_{1}$ (profit) from 2375 to 2600 and $f_{2}$ (quality) from 44.5 to 60 , respectively. Hence, one way to achieve the desired solution is via financing decisions.

TABLE I: INFORMATION TABLE OF EXAMPLE 1

\begin{tabular}{ccccc}
\hline \multirow{2}{*}{ Unit price } & \multirow{2}{*}{ Resource } & \multicolumn{2}{c}{ Technological coefficients } & \multirow{2}{*}{ No. of units } \\
\hline 30 & Nylon & 4 & $y=1$ & $b_{1}$ \\
40 & Velvet & 2 & 0 & $b_{2}$ \\
9.5 & Silver thread & 12 & 6 & $b_{3}$ \\
20 & Silk & 0 & 4 & $b_{4}$ \\
10 & Golden thread & 4 & 3 & $b_{5}$ \\
\hline
\end{tabular}

The problem of Example 1 is to derive the minimum extra budget which can achieve the desired point and determine the corresponding resource allocation. Solving the above problem, we can obtain the extra budget need $\widehat{B}=376$, productions $x_{1}=2$ and $x_{2}=6$. The corresponding resource allocation can be calculated as $b_{1}=8, b_{2}=40, b_{3}=48, b_{4}=18$ and $b_{5}=32$. The corresponding profit and quality index are exactly equal to 2600 and 60 , respectively.

Besides financial aids, a VO can also achieve its desired goal through improving objective coefficients of a system, e.g. economics of scale, electronic commerce, total quality management (TQM) and eliminating middle agencies. In this situation, a company should consider the unit improving cost of each objective coefficient and determine the optimal budget allocation between the improving costs and production resources. Then, we can develop a MOP model with changeable objective coefficients as follows:

<Model 2: MOP with changeable objective coefficients> 


$$
\begin{gathered}
\min \hat{B} \\
\text { s.t. } \quad \sum_{j=1}^{m}\left(c_{i j}+\widehat{c}_{i j}\right) x_{i j} \geq f_{i}^{* *}(\boldsymbol{x}), \quad i=1, \ldots, n, \\
\boldsymbol{p}^{\prime} \boldsymbol{A} \boldsymbol{x}+\sum_{i=1}^{n} \sum_{j=1}^{m} p_{i j}^{c} \widehat{c}_{i j} \leq B+\widehat{B}, \quad \text { extra conditions for } p_{i j}^{c} \text { and } \widehat{c}_{i j}> \\
\boldsymbol{x} \geq \mathbf{0},
\end{gathered}
$$

where $p_{i j}^{c}$ denotes the unit upgrading cost with respect to the $j$ th product coefficient of the $i$ th objective function and $\widehat{c}_{i j}$ is the $j$ th upgrading product coefficient of the $i$ th objective function.

Example 2. Following the previous example of producing suits and dresses. If the company cannot borrow money from capital markets, but also hope to increase $f_{1}$ (profit) from 2375 to 2600 and $f_{2}$ (quality) from 44.5 to 60 , respectively. Another way is to improve its objective coefficients through possible strategies

\begin{tabular}{|c|c|c|c|c|c|c|}
\hline \multicolumn{2}{|c|}{ Objective coefficients } & \multirow{2}{*}{ Unit price } & \multirow{2}{*}{ Resource } & \multicolumn{2}{|c|}{ Technological coefficients } & \multirow[b]{2}{*}{ No. of units } \\
\hline$x=1$ & $y=1$ & & & $x=1$ & $y=1$ & \\
\hline $400(\$ 0.200)$ & $300(\$ 0.289)$ & 30 & Nylon & 4 & 0 & $b_{1}$ \\
\hline \multirow[t]{4}{*}{$6(\$ 2.225)$} & $8(\$ 2.487)$ & 40 & Velvet & 2 & 6 & $b_{2}$ \\
\hline & & 9.5 & Silver thread & 12 & 4 & $b_{3}$ \\
\hline & & 20 & Silk & 0 & 3 & $b_{4}$ \\
\hline & & 10 & Golden thread & 4 & 4 & $b_{5}$ \\
\hline
\end{tabular}
or technologies. Therefore, we assume the unit improving costs of the objective coefficients are $\$ 0.200$, $\$ 0.289, \$ 2.225$ and $\$ 2.487$, respectively, as shown in Table 2 .

TABLE II: INFORMATION TABLE OF EXAMPLE 2

Solving the above problem, we can obtain the extra budget $\widehat{B}=0$. The result means that no extra budget is needed for achieving the desired point. Then, we can also derive $x_{1}=4.43, x_{2}=2.70, \widehat{c}_{11}=3.51$, $\widehat{c}_{12}=0.18, \widehat{c}_{21}=1.44$, and $\widehat{c}_{22}=2.00$. In addition, the corresponding resource allocation can be assigned as $b_{1}=17.72, b_{2}=25.06, b_{3}=17.72, b_{4}=8.10, b_{5}=28.52$, profit $=2600$ and quality index $=60$.

The last situation discussed here is that a VO may expand its outcome space through upgrading technology coefficients of a system. For example, a VO can adopt the business process reengineering (BPR), new information technologies or enterprise resource management (ERP) to increase the production efficiency, i.e., upgrading technology coefficients. Hence, we can conceptualize the above description to formulate the following model:

<Model 3: MOP with Changeable technological coefficients >

$$
\begin{gathered}
\min \widehat{B} \\
\text { s.t. } \quad \sum_{j=1}^{m} c_{i j} x_{i j} \geq f_{i}^{* *}(\boldsymbol{x}), \quad i=1, \ldots, n, \\
\boldsymbol{p}^{\prime}(\boldsymbol{A}-\widehat{\boldsymbol{A}}) \boldsymbol{x}+\sum_{k=1}^{r} \sum_{j=1}^{m} p_{k j}^{a} \widehat{a}_{k j} \leq B+\widehat{B},<\text { extra conditions for } p_{i j}^{a} \text { and } \widehat{a}_{i j}> \\
\boldsymbol{x} \geq \mathbf{0},
\end{gathered}
$$

where $\hat{\boldsymbol{A}}=[\hat{a}]_{k j}$ is the upgrading technological coefficient matrix and $p_{k j}^{a}$ is the unit upgrading cost with respect to the $j$ th technology coefficient of the $k$ th constraint.

\section{Discussions and Conclusions}

In this paper, we develop three MOP models for decision-makers to design or plan a system. The first model retains the original parameters of objective and technological coefficients but only considers expanding the available budget. The second model enables objective functions to be changeable so that a 
system can improve its objective coefficient to achieve the desired point. The last model considers the changeability of the technological coefficients of a system. Therefore, the desired point can be achieved through updating its technological coefficients.

Traditional MOP problems focus on the optimization within a system. However, the characteristics of VOs enable their systems to be re-designed or re-shaped to optimally perform. Hence, we should solve traditional MOP problems from a normative model to a prescriptive model. In this paper, we proposed three kinds of MOP with changeable parameters, i.e. budget, objective coefficients and technological coefficients, to help the decision-makers of VOs to achieve their desired points.

\section{References}

[1] Camarintha-Matos L. M., Afsarmanesh, H. and Ollis, M. (2005). Virtual Organizations: Systems and Practices. Springer.

[2] Charnes, A. and Cooper, W. W. (1961). Management models and industrial applications of linear programming. Wiley \& Sons: NY.

[3] ChiangLin, C. Y., Lai, T. C. and Yu, P. L. (2007). Linear programming models with changeable parameterstheoretical analysis on "taking loss at the ordering time and making profit at the delivery time. International Journal of Information Technology \& Decision Making 6 (4), 577-589.

[4] Chen, K.C., and Tzeng, G.H. (2009). Perspective strategic alliances and resource allocation in supply chain systems through the De Novo programming approach, International Journal of Sustainable Strategic Management, 1(3), 320-339.

[5] Dantzig, G. B. (1951). Upper bounds, secondary constraints, and block triangularity in linear programming. Econometrica 23 (2), 174-183.

[6] Davidow W. H., and Malone, M. S., (1993). The virtual corporation: structuring and revitalizing the corporation for the 21st Century. Harper Collins Publishers: NY.

[7] Donovan, G. H. and Rideout, D. B. (2003). An integer programming model to optimize resource allocation for wildfire containment, Forest Science, 49 (2), 331-335.

[8] Hackman, S.T., and Platzman, L.K. (1990). Near optimal solution of generalized resource allocation problems with large capacities. Operations Research 38 (5), 902-910.

[9] Huang, J.J., Tzeng, G.H., and Ong, C.S. (2006). Choosing best alliance partners and allocating optimal alliance resources using the fuzzy multi-objective dummy programming model. Journal of Operational Research Society, 57(10), 1216-1223.

[10] Huang, J. J., Tzeng, G. H. and Ong, C. S. (2005). Motivation and resource allocation for strategic alliance through De Novo perspective. Mathematical and Computer Modeling. 41 (6-7), 711-721.

[11] Kantorovich, L. V. and T. C. Koopmans, Problems of Application of Optimization Methods in Industry, Swedish: Federation of Swedish Industries (1976).

[12] Kim, H., Ida, K. and Gen, M. (1993). A de novo approach for bicriteria 0-1 linear programming with interval coefficients under gub structure. Computers and Industrial Engineering 25 (1), 17-20.

[13] Lambrechts, F., Grieten, S., Bouwen, R. and Corthouts, F. (2009). Process consultancy revisited. Taking a'relational practice'prespective. Journal of Applied Behavioral Science 45 (1), 39-58.

[14] Lee, E. S. (1994). On fuzzy de novo programming, in: fuzzy optimization: recent advances, edited by Delgado, M., Kacprzyk, J., Verdegay, J. L. and Vila, M. A. (Physica-Verlag, Heidelberg), 33-44.

[15] Li, H, L. and Yu, P. L. (1994). Optimal competence set expansion using deduction graph. Journal of Optimization Theory and Applications, 80 (1), 75-91.

[16] Li, R. J. and Lee, E. S (1990a). Multicriteria de novo programming with fuzzy parameters. Computers and Mathematics with Applications 19 (1), 13-20.

[17] Li, R. J. and Lee, E. S. (1990b). Fuzzy approaches to multicriteria de novo programs. Journal of Mathematical Analysis and Applications 153 (1), 97-111. 